\title{
On the Use of Time-Limited Information for Maintenance Decision Support: A Predictive Approach under Maintenance Constraints
}

\author{
E. Khoury, ${ }^{1}$ E. Deloux, ${ }^{1}$ A. Grall, ${ }^{1}$ and C. Bérenguer ${ }^{2}$ \\ ${ }^{1}$ Institut Charles Delaunay, Université de technologie de Troyes, STMR UMR CNRS 6279, 12 rue Marie Curie, CS 42060, \\ 10004 Troyes Cedex, France \\ ${ }^{2}$ Gipsa-lab, Control Systems Department, CNRS, Grenoble Institute of Technology, 11 rue des Mathématiques, \\ BP 46, 38402 Saint Martin d'Hères Cedex, France \\ Correspondence should be addressed to E. Khoury; elias.y.khoury@gmail.com
}

Received 14 March 2013; Revised 16 August 2013; Accepted 25 August 2013

Academic Editor: A. Elías-Zúñiga

Copyright (C) 2013 E. Khoury et al. This is an open access article distributed under the Creative Commons Attribution License, which permits unrestricted use, distribution, and reproduction in any medium, provided the original work is properly cited.

\begin{abstract}
This paper deals with a gradually deteriorating system operating under an uncertain environment whose state is only known on a finite rolling horizon. As such, the system is subject to constraints. Maintenance actions can only be planned at imposed times called maintenance opportunities that are available on a limited visibility horizon. This system can, for example, be a commercial vehicle with a monitored critical component that can be maintained only in some specific workshops. Based on the considered system, we aim to use the monitoring data and the time-limited information for maintenance decision support in order to reduce its costs. We propose two predictive maintenance policies based, respectively, on cost and reliability criteria. Classical age-based and condition-based policies are considered as benchmarks. The performance assessment shows the value of the different types of information and the best way to use them in maintenance decision making.
\end{abstract}

\section{Introduction}

The maintenance represents a substantial portion of the total life cycle cost of many systems. It becomes a very important aspect in the highly competitive industrial context. In many fields, such as transport (e.g., automotive and aeronautics), energy (e.g., nuclear facilities and petrochemistry), and critical infrastructures (e.g., roads and bridges), the interest of decision making in maintenance has increased in order to reduce the associated cost and/or improve the durability and the reliability of systems. Intensive researches have been achieved producing many models for the optimization of its scheduling [1-3]. In this work, we mainly focus on the transport field.

Many developments have been done in condition monitoring which provides information about the actual state of a deteriorating system. In particular, innovative technologies are implemented to some commercial vehicles in order to increase their competitiveness. With a better knowledge of the component's lifetime, it is possible to followup its useful life in order to replace it at the "optimal" time. It is less costly and allows to obviate a wrong use which causes some environmental damages.

The evolution to failure of a monitored component can then be assessed and described through a deterioration model to be used in the maintenance decision making. However, the deterioration behavior can be impacted by the prevailing environmental conditions [4] such as the usage conditions which can be considered as "covariates." In this case, the environment state should also be taken into consideration in the maintenance decision making process.

This paper investigates the problem of online maintenance decision making on a finite horizon in a predictive maintenance framework with constraints $[5,6]$. Preventive maintenance actions can only be planed at some specific times called maintenance opportunities. Information about the system state and the usage conditions are time limited. In particular, we consider the case of a commercial vehicle 
with a critical monitored component. For this case, the time is replaced by the running distance. The maintenance opportunities are specific positions corresponding to available workshops. The temperature, the type of road, and the load can be considered as covariates.

The efficiency of a maintenance strategy depends on the ability of the decision making process to adapt to changes in the system state and its environment. Therefore, the choice of types of information and the way to integrate them decide directly the performance of the maintenance policy. The latter should take into account a large number of information of different natures (e.g., deterioration level, environment state, and maintenance constraints) that may sometimes be contradictory [7]. The decision can then be very complex and sometimes inefficient. The more "useful" information is considered, the better the decision is. Thus, it is necessary to build indicators for maintenance decision support that integrate the maximum of relevant information.

For that purpose, maintenance strategies have been developed in the literature considering essentially the ConditionBased Maintenance (CBM) approach and recently the predictive approach. In the CBM approach, maintenance operations are scheduled according to health indicators, performance, or behavior of the monitored system. It is then expected to lead to better decisions than the age-based approach widely used in industry [8]. The CBM approach considers the current state of the system in the decision process; it is usually difficult to integrate all the useful information. In some works, such as in $[9,10]$, the operating environment state is considered in the decision process. In [11], possible changes in the deterioration process are taken into account. However, the CBM approach does not consider specific knowledge about some future characteristics. The latter is used in a mean way to optimize the parameters of the maintenance strategy. Actually, it is possible to know, in some cases, the specific future usage of the system and to "predict" a future characteristic. This prediction can be used for the decision making which should improve it. This prediction is called prognostic. The combination of the CBM and the prognostic leads to the predictive approach.

The prognostic is quite a new area of interest. It started to be brought up by the modal analysis community [2]. In a general way, it is defined as the prediction of the future state or characteristic of the system such as the remaining useful lifetime (RUL) [12, 13]. The RUL can integrate different information such as the deterioration level, the future covariates states if known, and so forth, and it can be used as a decision support tool in maintenance.

In this context, this paper deals with a maintenance study taking into account jointly two main problems: (i) the choice of a relevant maintenance decision structure that takes into account specific constraints related to maintenance opportunities on a finite rolling horizon, and (ii) the choice of the information to be used in the decision and the best way to integrate it. To address this problem, we develop two predictive maintenance strategies using the available information (including the covariates) differently, and we compare them with age-based and condition-based benchmark strategies.
The remainder of this paper is organized as follows. The first part is devoted to the system description and the assumptions. Specifically, we present the deterioration model and the maintenance constraints. In Section 3, we develop two proposed predictive maintenance policies and the assessment of the remaining useful lifetime. Then, the performance of the different policies is assessed through numerical examples considering various cases.

\section{System Description: Deterioration Modeling and Maintenance Constraints}

\subsection{Deterioration Model}

2.1.1. Basic Deterioration Model. Consider a gradually deteriorating system whose state can be summarized by a scalar random variable $X(t)$. The system is subject to a failure mechanism due to an excessive deterioration level " $L$ " (Figure 1). It is directly, perfectly, and continuously monitored, that is, its deterioration is completely observable at any moment [1]. The deterioration is assumed to be monotonously increasing over time $[14,15]$. The Gamma process is considered to describe its evolution.

The Gamma process is a stochastic process with independent, nonnegative increments having a Gamma distribution with an identical scale parameter. As far as the authors know, Abdel-Hameed [16] was the first to propose the Gamma process as a proper model for deterioration occurring randomly in time. An advantage of modeling deterioration through a Gamma is that the required mathematical calculations are relatively straightforward. The Gamma process is suitable to model gradual damage monotonically accumulating over time $[14,17]$ in a sequence of tiny increments, such as wear, fatigue, corrosion, crack growth, erosion, consumption, creep, swell, and degrading health index.

If $X(t)$ is a Gamma process with shape parameter $\alpha(t)$ and scale parameter $\beta$ then

(1) $X(0)=0$ (with a probability equal to 1 ),

(2) $X\left(t_{2}\right)-X\left(t_{1}\right) \sim \mathrm{Ga}\left(\alpha\left(t_{2}\right)-\alpha\left(t_{1}\right), \beta\right)$,

(3) $X(t)$ has independent increments,

where $\mathrm{Ga}(a, b)$ denotes a Gamma law with shape parameter $a$ and scale parameter $b$.

For a Gamma distributed random variable $Y$ with parameters $\alpha(t)$ and $\beta: Y \sim \mathrm{Ga}(\alpha(t), \beta)$, the probability density function $f(y)$ and the cumulative distribution function $F(y)$ are, for $y>0$, given by

$$
\begin{aligned}
& f(y)=\frac{(1 / \beta)^{\alpha(t)}}{\Gamma(\alpha(t))} y^{\alpha-1} e^{-y / \beta} \\
& F(y)=1-\frac{\Gamma(\alpha(t), y / \beta)}{\Gamma(\alpha(t))},
\end{aligned}
$$

where $\Gamma(a, z)=\int_{z}^{\infty} u^{a-1} e^{-u} d u$ and $\Gamma(a)=\int_{0}^{\infty} u^{a-1} e^{-u} d u$.

Over a time interval of length $t$, the average deterioration speed rate of a Gamma process is $\alpha(t) \cdot \beta$ and its variance 


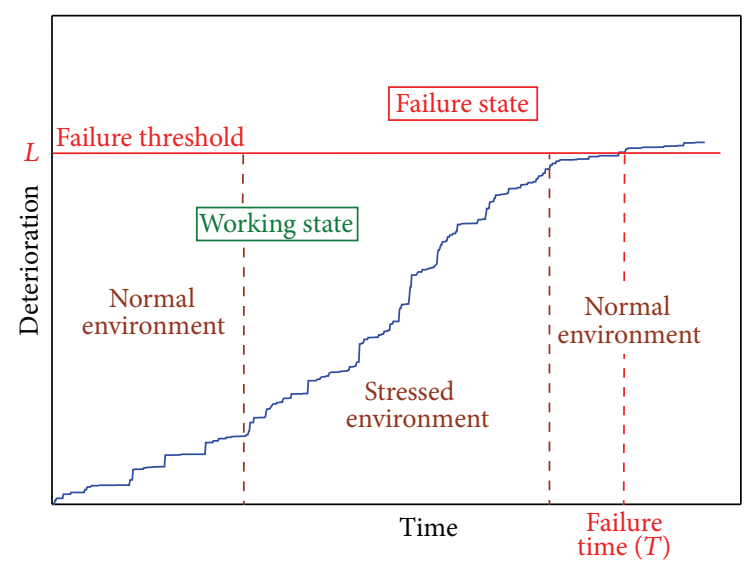

FIGURE 1: Deterioration evolution to failure.

$\alpha(t) \cdot \beta^{2}$. The choice of $\alpha$ and $\beta$ allows one to model various deterioration behaviors from almost deterministic to very chaotic. $\alpha$ and $\beta$ can be estimated using classical statistical methods given the deterioration data (the maximum likelihood method, the moments method, Bayesian statistics method, etc.). One can see [18] for more details.

If $\alpha(t)$ is a linear function of time that is $\alpha(t)=\alpha \cdot t$, the Gamma process is a stationary (also called homogeneous) Gamma process. In this case, the expected deterioration is linear over time. The stationarity of the Gamma process basically follows from the property that increments are independent and have the same type of distribution as their sum. A random variable following a Gamma law with parameters $\alpha$ and $\beta$ can be written as the sum of $n$ variables following a Gamma law with parameters $\alpha / n$ and $\beta$ [18].

2.1.2. Environment Effect. The system is operating under a fluctuant environment (covariates) impacting its deterioration. The covariates are additional explanatory variables such as the operating environment (e.g., vibration, temperature, and air humidity), the conditions of use (e.g., load and production rate), and underlying physical phenomena explaining the evolution of system state. The covariates impact on deterioration is considered in many models in the literature and in several ways. One can divide the different impacts on three main types. The first type considers a punctual impact of the covariates on the deterioration. In this case, a covariate change causes an additional deterioration jump. This type of impacts is considered in $[19,20]$. The second type considers continuous temporal impact of covariates. Usually, the parameters of the deterioration process depend on the environment of use $[21,22]$. The third type of impact is not on the deterioration directly but on the failure of the system either on the deterioration failure threshold [23] or the type of failure [24].

In this work, the second type is considered. We assume that the system operates under a two-stage environment: "normal" and "stressed", and the deterioration follows a homogeneous Gamma process for each of the environment states. Let $\left(\alpha_{1} \cdot t, \beta_{1}\right)$ and $\left(\alpha_{2} \cdot t, \beta_{2}\right)$ denote the couples of shape and scale parameters for, respectively, the "normal" and

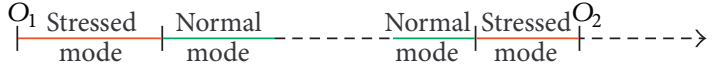

FIgURE 2: A visibility horizon example. $O_{i}$ represents the $i$ th opportunity.

the "stressed" states. The average deterioration speed rate is higher under the stressed environment: $\alpha_{1} \cdot \beta_{1}<\alpha_{2} \cdot \beta_{2}$.

\subsection{Maintenance Constraints}

2.2.1. Constraints on the Maintenance Dates. The system is subject to constraints; maintenance actions cannot be planned at any time [25] but only at fixed maintenance opportunities. This kind of constraint can exist in many fields such as aeronautics field, nuclear facilities, off-shore plants, and rail, and for many reasons such as availability constraints, technical constraints, the necessity of specialized maintenance team, or equipments.

2.2.2. Limited Visibility Horizon. The maintenance opportunities are known in advance on a sliding finite horizon. When the system evolves in time, the knowledge evolves too and it is available on an extended horizon. Hence, it is a "finite rolling visibility horizon." Herein, we assume that at least two maintenance opportunities are available on the visibility horizon, and the operating environment, that is, the covariate is known, at least, until the second opportunity (Figure 2).

2.2.3. Specification of the Maintenance Problem. In the considered context, the challenge is to schedule maintenance tasks in the available opportunities, based on the time-limited information, in order to reduce their cost. Actually, when a failure occurs, the system is repaired resulting in a corrective $\operatorname{cost} C_{c}$ higher than the preventive cost $C_{p}$, and a cost relative to the time of failure $C_{d}(t)$ which can be, for example, relative to the downtime duration $t_{d}: C_{d}(t)=C_{d} \cdot t_{d}$.

The goal is then to decide at each opportunity $O_{i}$ between the following options:

(1) taking this opportunity $O_{i}$,

(2) waiting for the next opportunity on the visibility horizon $O_{i+1}$ to update the decision with the new available information.

In this framework, one has to find a tradeoff between maintaining too early at the first opportunity (i.e., loosing useful lifetime) and scheduling a maintenance too late at the second opportunity or later (i.e., risking a failure and the associated costs).

To this end, we propose in Section 3 two predictive maintenance strategies. In the predictive approach, the maintenance decision is based on the forecast of the future evolution of the system called prognostic. The prognostic has several definitions [13]; however, in a general way it is defined as the prediction of the future state or characteristic of the system such as the remaining useful lifetime (RUL) [12]. 


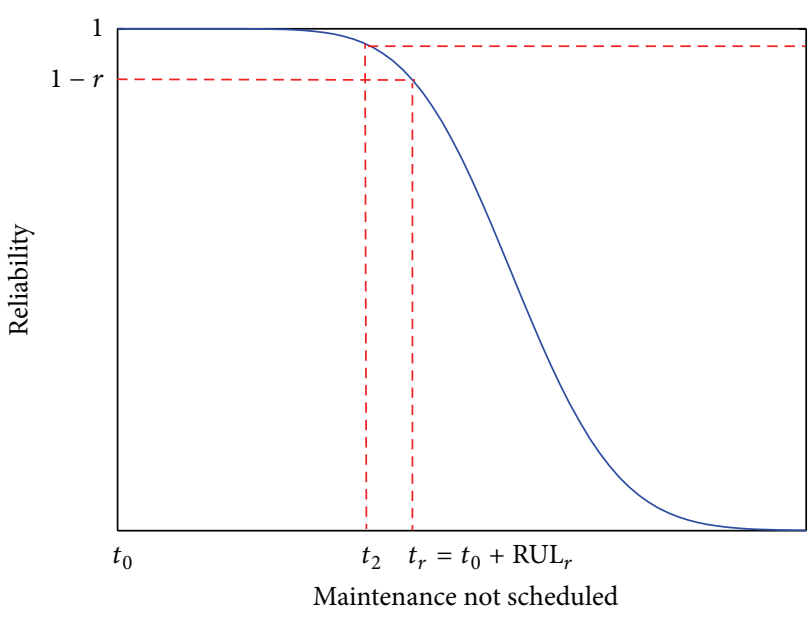

(a) $r_{2}<r$

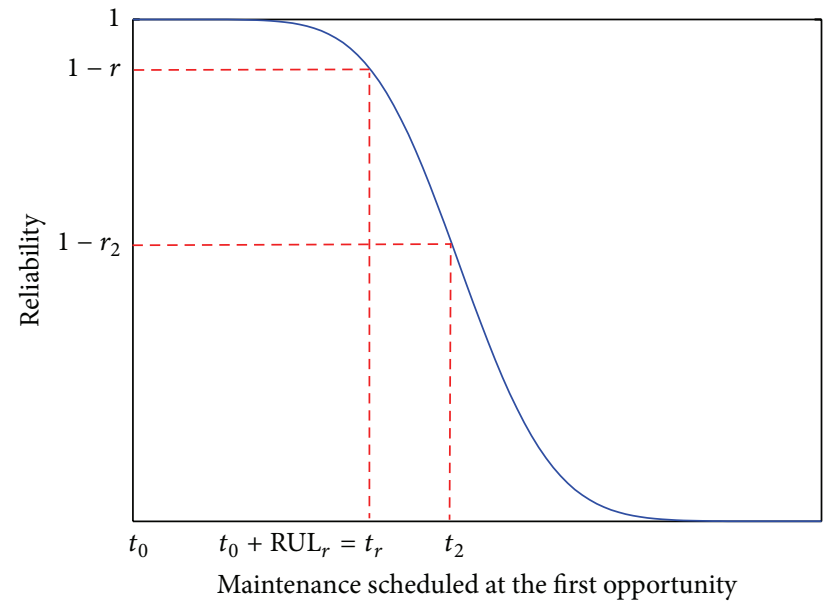

(b) $r_{2}>r$

Figure 3: Maintenance decision making for the $R$-based policy.

The RUL is the "duration" left for a system before it fails. It can be considered as a simple duration with a given probability of failure, a confidence interval, or more generally a stochastic variable with a probability distribution. The third case is considered in this work. One can assess the failure time in order to find the RUL, since it is the duration between the failure time and the current time. Actually, given that the system survived until $t_{0}$, the probability that it fails before a duration $T_{0}$ is the probability that the failure occurs before $t=t_{0}+T_{0}$. Let $T$ be the time of failure and $R\left(t \mid X\left(t_{0}\right)\right)$ the conditional reliability, and $P\left(\right.$ RUL $\left.<T_{0} \mid X\left(t_{0}\right)\right)=P(T<$ $\left.t_{0}+T_{0} \mid X\left(t_{0}\right)\right)=1-R\left(t \mid X\left(t_{0}\right)\right)$. Assessing the RUL (as a stochastic variable) is, thus, done through the assessment of the (conditional) reliability.

The RUL assessment can integrate different information such as the deterioration level and the future covariates states. It can be used as a decision support tool in maintenance. Hence, this approach integrates all the available information, and the decision is adapted to the current and the future state of the system. It is then a dynamically adaptive approach.

\section{Predictive Maintenance Policies}

This section is devoted to the development of the proposed predictive maintenance policies. We describe the reliabilitybased policy with the RUL assessment and the cost-based policy, respectively, in Sections 3.1 and 3.2. Then we present the case of failure between opportunities.

\subsection{Reliability-Based Policy}

3.1.1. Policy Description. The reliability-based policy, denoted $R$-based, is a predictive adaptive one integrating the prognostic in the maintenance decision making. Actually, we consider in this policy that the probability of failure should not exceed a threshold $r$ (to be optimized), that is, the conditional reliability of the system must be greater than $1-r$ at any moment. For this reason, we examine the first two maintenance opportunities on the visibility horizon. The probability of failure at each opportunity can be found using the RUL distribution (or the reliability function). If the probability of failure at the second opportunity is greater than $r$, this means that the system reliability constraint will be violated before reaching the second opportunity. Hence, a preventive replacement is scheduled at the first one.

The RUL is used directly in the decision making structure. Let $\mathrm{RUL}_{r}$ denote the remaining lifetime corresponding to the probability of survival $1-r$ and $t_{r}$ the corresponding (estimated) time of failure: $P\left(T<t_{r} \mid X\left(t_{0}\right)\right)=r$ and $\mathrm{RUL}_{r}=t_{r}-t_{0}$. The $R$-based policy is equivalent to compare the remaining lifetime $\mathrm{RUL}_{r}$ (or the expected time of failure $t_{r}=t_{0}+\mathrm{RUL}_{r}$ ) to the time of the second opportunity $t_{2}$. Hence, the maintenance decision is as follows.

(i) If the probability of failure $r_{2}$ at the second opportunity is lower than $r$ (Figure 3(a)), this means $t_{2}<t_{r}$ and the remaining lifetime is enough to reach the second opportunity with a good level of confidence, that is, the system can survive till $t_{2}$ with the probability $1-r_{2}>1-r$.

(ii) Otherwise (Figure 3(b)), the remaining lifetime (with probability of survival $1-r$ ) is not enough to reach the second opportunity. Maintenance operations should then be scheduled at the first one.

3.1.2. RUL Assessment. In the considered case of the "failure threshold model," the assessment of the reliability consists in finding the probability that the system deterioration $X(t)$ does not exceed the failure threshold $L$ at a given time $t$. Consider a visibility horizon of two maintenance opportunities at, respectively, $t_{i}$ and $t_{i+1}$. The current position $t_{0}$ is considered to be equal to $t_{i}$; otherwise, the assessment of the reliability would be similar. Several environment changes can occur between the opportunities. This case is discussed at the end of this section. For convenience consideration, two cases 


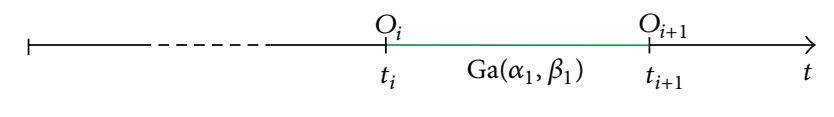

FIGURE 4: Opportunities without environment change.

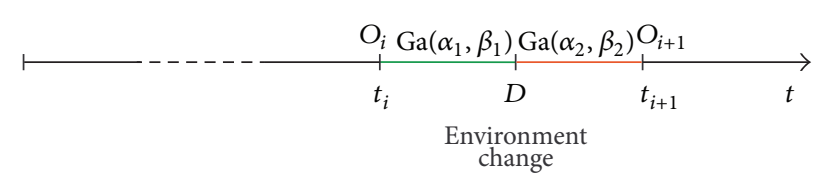

FIGURE 5: Opportunities with environment change.

of environment changes between opportunities are taken into account as follows.

(1) The environment state remains the same between the two opportunities (Figure 4). We refer to this case by "opportunities without environment change."

(2) There is an environment change (at $D$ ) between the opportunities (Figure 5). We refer to this case by "opportunities with environment change."

Let $R_{x_{0}}(t)$ denote the conditional reliability $R\left(t \mid X\left(t_{0}\right)\right)$ at $t$ given that the deterioration level at $t_{0}$ is $x_{0}, f(t)$ and $F(t)$, respectively, the probability distribution function (pdf) and the cumulative distribution function (cdf) relative to the failure instant. If $X(t) \sim \mathrm{Ga}(a \cdot t, b)$, we note $F_{\Gamma}(x, a, b)=$ $P(X(t) \leq x)$ its cdf and $f_{\Gamma}$ its pdf. $T$ is the time of failure.

Opportunities without Environment Change (Figure 4). If the environment state is normal

$$
\begin{aligned}
R_{x_{0}}(t) & =P\left(T>t \mid X\left(t_{i}\right)=x_{0}\right) \\
& =P\left(X(t)<L \mid X\left(t_{i}\right)=x_{0}\right) \\
& =P\left(X(t)-x_{0}<L-x_{0} \mid X\left(t_{i}\right)=x_{0}\right) \\
& =F_{\Gamma}\left(L-x_{0}, \alpha_{1} \cdot\left(t-t_{i}\right), \beta_{1}\right) .
\end{aligned}
$$

If the environment is stressed, $\left(\alpha_{1}, \beta_{1}\right)$ should be replaced by $\left(\alpha_{2}, \beta_{2}\right)$.

Opportunities with Environment Change (Figure 5). If the environment state is first normal, then stressed

$$
R_{x_{0}}(t)=R_{x_{0}, 1}(t) \cdot \mathbf{1}_{\{t \leqslant D\}}+R_{x_{0}, 2}(t) \cdot \mathbf{1}_{\{t>D\}},
$$

where

$$
\begin{gathered}
R_{x_{0}, 1}(t)=F_{\Gamma}\left(L-x_{0}, \alpha_{1} \cdot\left(t-t_{i}\right), \beta_{1}\right), \\
R_{x_{0}, 2}(t)=P\left(T>t \mid X\left(t_{i}\right)=x_{0}\right)
\end{gathered}
$$

$$
\begin{aligned}
= & \int_{x_{0}}^{L} P(T>t \mid X(D)=u) \\
& \cdot P\left(X(D)=u \mid X\left(t_{i}\right)=x_{0}\right) d u
\end{aligned}
$$$$
=\int_{x_{0}}^{L} F_{\Gamma}\left(L-u, \alpha_{2} \cdot(t-D), \beta_{2}\right)
$$$$
\cdot f_{\Gamma}\left(u-x_{0}, \alpha_{1} \cdot\left(D-t_{i}\right), \beta_{1}\right) d u .
$$

If the environment state is first stressed, then normal $\left(\alpha_{1}, \beta_{1}\right)$ and $\left(\alpha_{2}, \beta_{2}\right)$ should be inverted.

In the case of several environment changes between the opportunities, the assessment of the reliability would be similar. Consider $n$ environment changes between $t_{i}$ and $t \leqslant$ $\left.t_{i+1}\right)$ at, respectively, $t_{i 1}, t_{i 2}, \ldots, t_{i n}$. The deterioration jump between $t_{i}$ and $t$ is the sum of the jumps between $t_{i}$ and $t_{i 1}$, $t_{i 1}$ and $t_{i 2}, \ldots, t_{i n}$ and $t$; these jumps are Gamma distributed. The convolution of their probability distribution functions is the one of the total jump between $t_{i}$ and $t$. One can then find the reliability by integrating the deterioration pdf. Several methods can be used for this computation, in our work we use Monte Carlo simulations to assess the reliability in this case.

3.2. Cost-Based Policy. The cost-based policy, denoted CIbased, is also a predictive adaptive policy. However, the prognostic is integrated in the maintenance decision making different from the $R$-based one. Since the aim is to reduce the maintenance cost, one should choose the opportunity that would lead to the least cost. For that, we consider a cost indicator $\mathrm{CI}$ as a tool for maintenance decision support. $\mathrm{CI}$ is the long-run cost rate for the studied situation (current deterioration level, environment state, etc.) if the maintenance is scheduled in one of the opportunities. It represents how much it would cost on average if the maintenance is scheduled in a given opportunity.

Let $\mathrm{CI}_{i}$ and $\mathrm{CI}_{i+1}$ denote the cost indicators corresponding to the first and the second opportunities, respectively. If $\mathrm{CI}_{i}<\mathrm{CI}_{i+1}$ (Figure 6(a)) this means that, on average, it will be more expensive to schedule the maintenance at the second opportunity than at the first one. Hence, a preventive replacement is scheduled at the first opportunity. Otherwise, the decision would be not to schedule the maintenance at the first opportunity nor at the second (Figure 6(b)).

Actually, when the first opportunity is overtaken, a new one appears in the visibility horizon since it is a rolling one on which there are, at least, two available opportunities. We compare then the cost indicators for both opportunities in the same way. The decision making process ends when the maintenance task is scheduled or when the system fails. Note that the cost indicator is not the real cost of the maintenance, it is only a tool for decision support.

Consider that the system is at the first opportunity $\left(t_{0}=\right.$ $t_{i}$ ), and it is still working; otherwise, a corrective maintenance action would have been performed. If this first opportunity is 


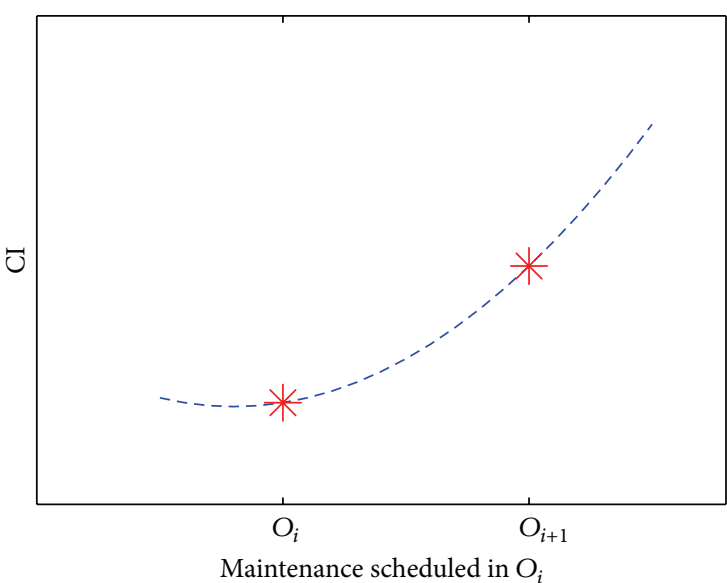

(a) $\mathrm{CI}_{i}<\mathrm{CI}_{i+1}$

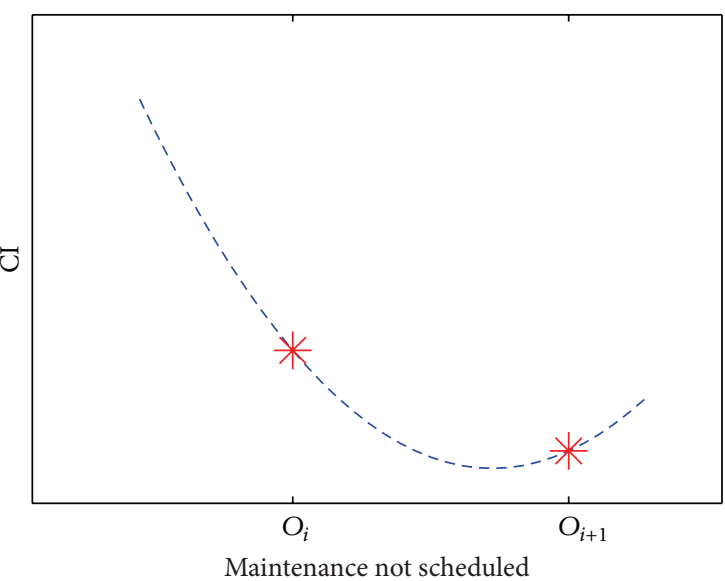

(b) $\mathrm{CI}_{i}>\mathrm{CI}_{i+1}$

FIGURE 6: Maintenance decision making for the CI-based policy.

chosen to schedule the maintenance, it would be a preventive replacement. Hence

$$
\mathrm{CI}_{i}=\frac{C_{p}}{t_{i}} .
$$

$\mathrm{CI}_{i+1}$ is defined as

$$
\mathrm{CI}_{i+1}=\frac{C}{D V}
$$

where $C$ and $D V$ are, respectively, the maintenance cost and the mean uptime.

Consider

$$
\begin{aligned}
C= & C_{p} \cdot P\left(T>t_{i+1} \mid X\left(t_{i}\right)=x_{0}\right) \\
& +C_{c} \cdot P\left(T \leq t_{i+1} \mid X\left(t_{i}\right)=x_{0}\right)+E\left[C_{d}(T)\right] \\
= & C_{p} \cdot R\left(t_{i+1} \mid X\left(t_{i}\right)=x_{0}\right) \\
& +C_{c} \cdot F\left(t_{i+1} \mid X\left(t_{i}\right)=x_{0}\right)+E\left[C_{d}(T)\right] .
\end{aligned}
$$

$D V$ is given by

$$
\begin{aligned}
D V & =t_{i+1}-\int_{t_{i}}^{t_{i+1}}\left(t_{i+1}-t\right) \cdot f\left(t \mid X\left(t_{i}\right)=x_{0}\right) d t \\
& =t_{i+1}-\int_{t_{i}}^{t_{i+1}} F\left(t \mid X\left(t_{i}\right)=x_{0}\right) d t \\
& =t_{i+1}-I_{1}-I_{2} .
\end{aligned}
$$

\section{Performance Assessment}

Consider now the particular case of a vehicle with a critical monitored component that can be maintained only in some specific workshops. The latter are the maintenance opportunities for this case. The normal/stressed environment can correspond, for example, to the empty/loaded vehicle. The deterioration is considered to be distance-dependant; hence, $t$ represents the running distance and not the time.
We assume that if a failure occurs, a maintenance team is shifted from the nearest workshop resulting in the cost $C_{d}(t)=C_{d} \cdot \Delta(t)$, where $\Delta(t)$ is the distance to the nearest workshop at position $t: \Delta(t)=\min \left\{t-t_{i}, t_{i+1}-t\right\}$. The development of the formulas using the expression of $\Delta(t)$ leads to

$$
\begin{aligned}
E\left[C_{d}(T)\right]=C_{d} \cdot( & -\int_{t_{i}}^{\left(t_{i}+t_{i+1}\right) / 2} F\left(t \mid X\left(t_{i}\right)=x_{0}\right) d t \\
& \left.+\int_{\left(t_{i}+t_{i+1}\right) / 2}^{t_{i+1}} F\left(t \mid X\left(t_{i}\right)=x_{0}\right) d t\right) \\
=C_{d} \cdot( & \left.-I_{1}+I_{2}\right),
\end{aligned}
$$

where $I_{1}=\int_{t_{i}}^{\left(t_{i}+t_{i+1} / 2\right)} F\left(t \mid X\left(t_{i}\right)=x_{0}\right) d t$ and $I_{2}=\int_{\left(t_{i}+t_{i+1} / 2\right)}^{t_{i+1}}$ $F\left(t \mid X\left(t_{i}\right)=x_{0}\right) d t$.

The expressions of $I_{1}$ and $I_{2}$ can be developed by considering the reliability expressions presented previously. The cost indicators become

$$
\begin{gathered}
\mathrm{CI}_{i}=\frac{C_{p}}{t_{i}}, \\
\mathrm{CI}_{i+1}=\frac{C_{p} \cdot R\left(t_{i+1} \mid X\left(t_{i}\right)=x_{0}\right)}{t_{i+1}+I_{1}+I_{2}} \\
+\frac{C_{c} \cdot F\left(t_{i+1} \mid X\left(t_{i}\right)=x_{0}\right)}{t_{i+1}+I_{1}+I_{2}} \\
+\frac{C_{d} \cdot\left(-I_{1}+I_{2}\right)}{t_{i+1}+I_{1}+I_{2}} .
\end{gathered}
$$

In order to assess the performance of the proposed predictive strategies in Sections 3.1 and 3.2, we develop, next, classical benchmark policies adapted to the vehicle case. Then, we present some numerical results.

4.1. Benchmark Policies. We consider the age-based and the condition-based maintenance approaches and propose two benchmark strategies adapted to our case study. 
4.1.1. Age-Based Maintenance. The age-based maintenance approach $[26,27]$ is the most common approach, it is explored extensively in the literature. It is based essentially on the systematic preventive maintenance taking only into account the age of the system. It can be based solely on the failures history and the experience of the maintenance team, the reason for which it is widely applied in industry [26].

The age-based approach is well adapted to lifetime models. Its application does not require any knowledge about the current state of the system (except its age) and does not include any monitoring information. Maintenance decisions are determined offline based on a priori knowledge. Despite the latest extensions $[26,28]$, this approach can be considered as static. It can lead to very early or very late interventions because it does not take into account neither the precise state of the system (deterioration level for example) nor the operating environment. Hence, in general, it is not efficient enough to meet the current industrial needs but it is robust and conservative.

In this work, an age-based policy is considered as a benchmark policy, it is adapted though to the studied case with the maintenance opportunities. If the system (vehicle for example) is still working, a preventive replacement is scheduled at the last maintenance opportunity before an age $T$ (a running distance for the vehicle case) to be optimized. The maintenance decision making is then only based on the age of the system and its binary (working/failure) state.

4.1.2. Condition-Based Maintenance. Multiple maintenance strategies are developed under the condition-based maintenance (CBM) approach [26], but it is still much less explored than the age-based one since it is more recent and request more effort to be applied. The CBM approach has proved to be efficient in terms of maintenance costs saving when the system deterioration can be monitored. Actually, in the CBM approach, maintenance operations are scheduled according to health indicators, performance, or behavior of the monitored system. It is then expected to lead to better decisions than the age-based approach [8]. Usually, one or many preventive replacement thresholds are considered [9], and when the deterioration exceeds a threshold (depending on the specific case or environment state) a preventive replacement is scheduled. This approach uses the precise state of the system in a dynamic way, but it is not completely adaptive. Actually, the CBM approach considers only the current state of the system in the decision process; it is usually difficult to integrate all the useful information. In some works, such as $[9,10]$, the operating environment state is considered in the decision process. However, the CBM approach doesn't consider the knowledge about some specific future characteristics, the information considered online is limited to the present.

Herein, we consider a condition-based policy such that, at each opportunity, if the deterioration level exceeds a fixed preventive replacement threshold $M$ (to be optimized), a preventive replacement is scheduled. Clearly, this policy is more adaptive than the age-based policy and should lead to a better maintenance decision. However, the decision may probably be improved by taking into account the future environment state (on the visibility horizon) and the predicted evolution of the system state as for the proposed predictive strategies.

4.2. Numerical Results. Depending on the considered approach, different information are integrated in the maintenance decision making, and the same information is used in two different ways within the predictive maintenance approach. It is expected that the predictive maintenance policies using more "useful" information, such as known/predicted future characteristics, would lead to better decision making than the condition-based approach which should be better than the age-based one that is the least adaptive. However, it would be interesting to quantify the performance difference between the three approaches but also within the predictive approach and to compare it in several cases such as different deterioration parameters, environment impact, and costs.

Given the considered economic context, we examine the expected long-run maintenance cost rate in several cases. Let $C(t)$ denote the real maintenance cost on a period (distance) of length $t$, the expected long-run cost rate is given by

$$
\lim _{t \rightarrow \infty} \frac{C(t)}{t}
$$

Therefore, we use Monte Carlo simulations such that the long-run cost rate is convergent. The parameters of the policies are optimized on the basis of the minimization of $C(t)$.

We also consider the mean distance till maintenance denoted MDTM as a performance indicator of the policies. Actually, the more the MTDM is high, the more maintenance stops are distant. The system, herein the vehicle, can run more which is usually more convenient.

In the following, we present four cases for which the length of the successive environment states and the distance between opportunities are simulated using uniform laws $U(40,80)$ and $U(25,40)$, respectively. The considered costs $\left(C_{c}, C_{d}\right.$ and $\left.C_{p}\right)$ are illustrative. In each case, the corrective $\operatorname{cost} C_{c}$ is set to 100 as a reference. The preventive cost $C_{p}$ and the failure costs $C_{d}$ are then considered to take small, medium, or high values compared to $C_{c}$. The failure threshold $L=100$. The rest of the model parameters and the costs for these cases are

Case 1. $\left(\alpha_{1}, \beta_{1}\right)=(0.5,0.5),\left(\alpha_{2}, \beta_{2}\right)=(0.75,0.5), C_{p}=90$ and $C_{d}$ varies between 1 and 150 .

Case 2. $\left(\alpha_{1}, \beta_{1}\right)=(0.5,0.5),\left(\alpha_{2}, \beta_{2}\right)=(0.75,0.5), C_{p}=25$ and $C_{d}$ varies between 1 and 150 .

Case 3. $\left(\alpha_{1}, \beta_{1}\right)=(0.5,0.5),\left(\alpha_{2}, \beta_{2}\right)=(0.75,0.5), C_{p}=10$, 25, 90 and $C_{d}$ varies between 1 and 150 .

Case 4. $\left(\alpha_{1}, \beta_{1}\right)=(0.5,0.5),\left(\alpha_{2}, \beta_{2}\right)=(0.75,0.5)$ or $\left(\alpha_{2}\right.$, $\left.\beta_{2}\right)=(1,0.75), C_{p}=25$ and $C_{d}$ varies between 1 and 150 . 


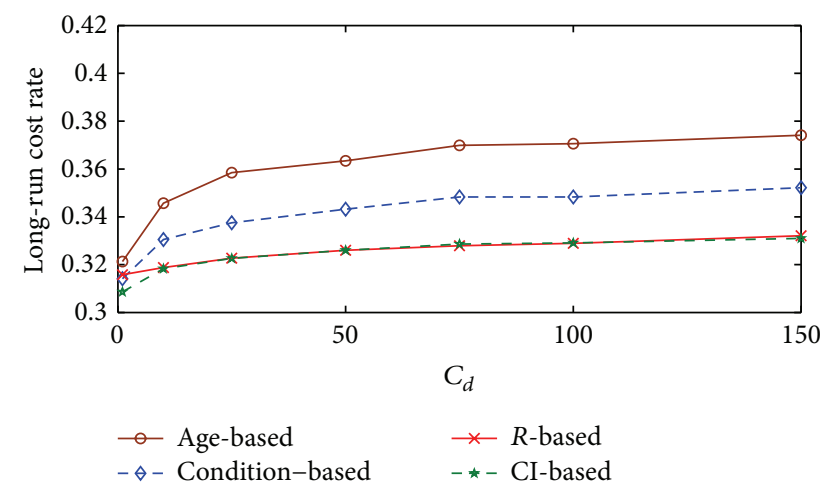

Figure 7: Case 1-Long-run cost rate variation depending on the maintenance policy and $C_{d}$.

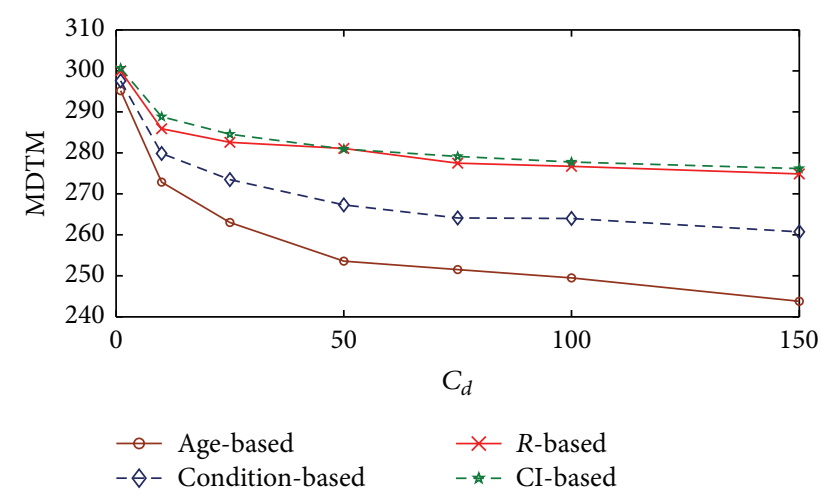

Figure 8: Case 1-Mean distance till maintenance (MDTM) depending on the maintenance policy and $C_{d}$.

We first consider the Case 1 and vary the cost $C_{d}$ relative to the downtime. The long-run cost rates for the four policies are presented in Figure 7.

As expected, the predictive policies (the $R$-based and the CI-based policies) lead to lower costs than the conditionbased and the age-based policies, however, they have almost the same performance. Compared to the age-based policy, the condition-based policy reduces the maintenance cost from around $2.5 \%$ to $6 \%$ while the two predictive policies (having almost the same performance) present a cost gain from around $4 \%$ to $11.5 \%$. The gain increases with the increase of $C_{d}$. Actually, when $C_{d}$ increases, a failure will cost more; hence, a better decision avoiding failures should reduce more the maintenance cost and thus lead to a higher gain.

We examine also another aspect of the performances of the maintenance policies: the mean distance till maintenance MDTM for the four policies and different values of $C_{d}$ (Figure 8 ). The MDTM is around 3.5\% to 6\% higher for the condition-based policy and $8.5 \%$ to $11 \%$ for the predictive policies compared to the age-based policy. This means that the adaptive policies, that is, the condition-based and the predictive policies allow the system to run longer with less maintenance costs than the static age-based policy.

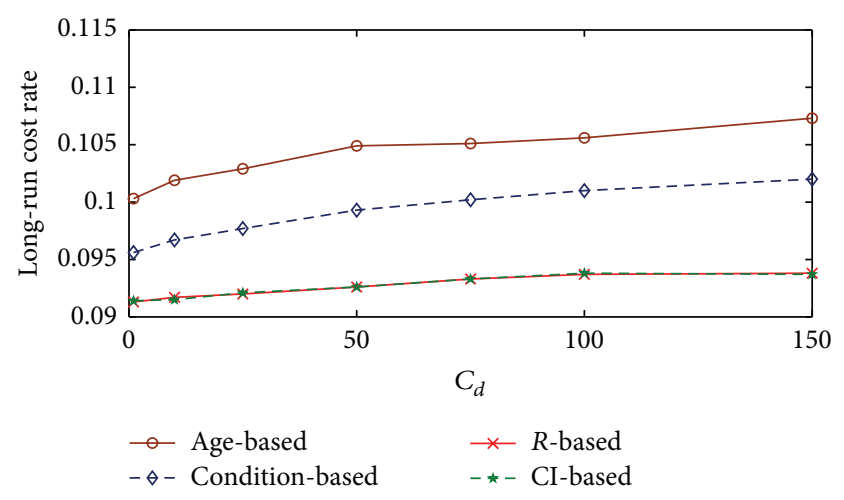

FIgURE 9: Case 2-Long-run cost rate variation depending on the maintenance policy and $C_{d}$.

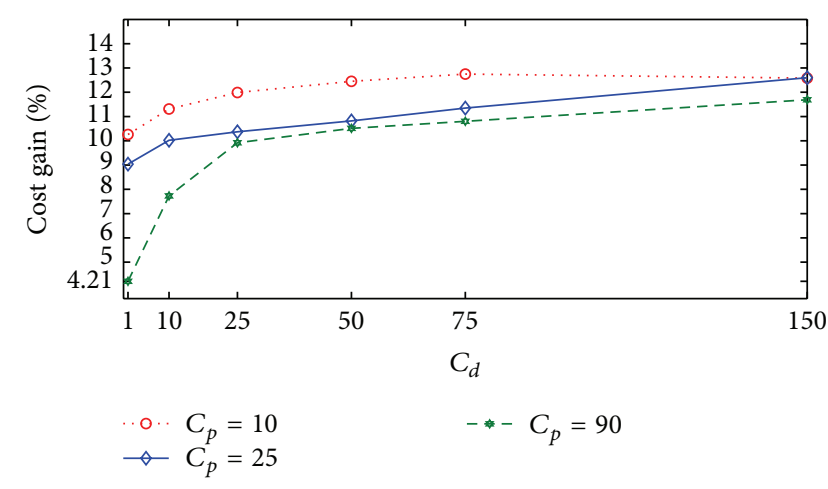

Figure 10: Case 3-The CI-based policy cost gain comparing to the age-based policy depending on $C_{p}$ and $C_{d}$.

Consider now the second case for which the preventive replacement cost is lower than in the first case. The longrun costs for the four policies depending on $C_{d}$ are presented in Figure 9. As in the first case, the predictive policies have almost the same performance and they lead to lower costs. Compared to the age-based policy, the condition based policy reduces the maintenance cost from around $4.5 \%$ to $5 \%$ while the predictive policies present a cost gain from around $9 \%$ to $13 \%$.

To assess the evolution of the cost gain depending on the different costs, we consider Case 3 and compute the gain of the CI-based policy (since both predictive policies lead to the same costs) compared to the age-based policy. Results are presented in Figure 10. For all the set of parameters, the gain increase with the increase of $C_{d}$ since the predictive policies avoid failures that would cost more when $C_{d}$ increases. The gain goes from around $4 \%$ to $12.5 \%$. It decreases in general when $C_{p} / C_{c}$ increases. Actually, for the same downtime cost $C_{d}$, when $C_{p} / C_{c}$ increases, that is, the preventive replacement cost $C_{p}$ is closer to the corrective replacement cost $C_{c}$, the difference between the replacement costs is lower, and hence choosing the one or the other would have a smaller impact on the total cost. A better decision would then lead to a lower cost gain. For the extreme case where $C_{c}=C_{p}$ and $C_{d}=0$, 
TABLE 1: Comparison between the CI-based and the $R$-based policies.

\begin{tabular}{lccccccc}
\hline Case & $C_{d}$ & $C_{\mathrm{CI}}$ & $C_{R}$ & $G_{\text {cost }}$ & MDTM $_{\mathrm{CI}}$ & MDTM $_{R}$ & $G_{\mathrm{MDTM}}$ \\
\hline 5 & 10 & 0.0331 & 0.0339 & $2.45 \%$ & 304.08 & 307.5931 \\
6 & 25 & 0.0332 & 0.0340 & $2.34 \%$ & 303.34 & 307.5734 \\
\hline
\end{tabular}

TABLE 2: Comparison between the CI-based and the R-based policies considering different costs between opportunities.

\begin{tabular}{lcccccccc}
\hline Case & Environment & Opportunities & $C_{c}$ & $C_{p} / C_{c}$ & $C_{d} / C_{c}$ & $C_{\mathrm{CI}}$ & $C_{R}$ & $C_{R}-C_{\mathrm{CI}} / C_{\mathrm{CI}}$ \\
\hline 7 & $U(40,80)$ & $U(25,40)$ & $U(80,100)$ & 0.1 & 0.1 & 0.0348 & 0.0328 & $-5.75 \%$ \\
8 & $U(70,90)$ & $U(50,70)$ & $U(80,100)$ & 0.1 & 0.1 & 0.0394 & 0.0376 & $-4.57 \%$ \\
9 & $U(70,90)$ & $U(50,70)$ & $U(90,100)$ & 0.1 & 0.1 & 0.0406 & 0.0396 & $-2.46 \%$ \\
10 & $U(90,100)$ & $U(70,80)$ & $U(90,100)$ & 0.1 & 0.1 & 0.0497 & 0.0496 & $-0.2 \%$ \\
11 & $U(90,100)$ & $U(70,80)$ & $U(90,100)$ & 0.9 & 0.1 & 0.4121 & 0.4136 & $+0.36 \%$ \\
\hline
\end{tabular}

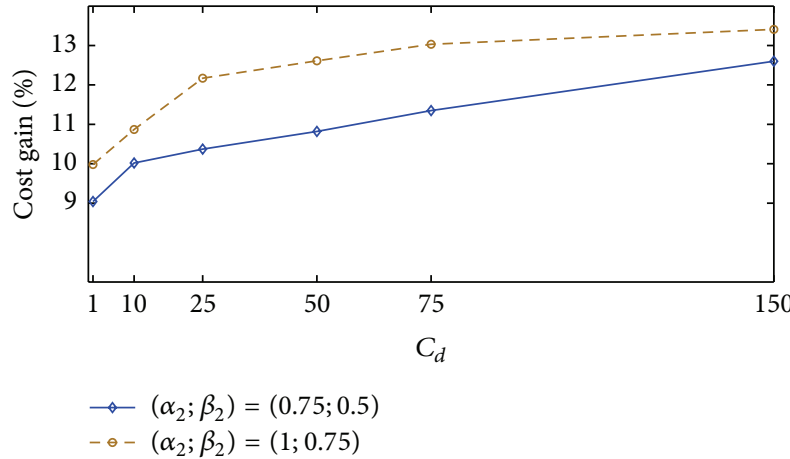

FIGURE 11: Case 4-The CI-based policy cost gain comparing to the age-based policy depending on the deterioration speed rate and $C_{d}$.

only corrective maintenance would be done for both policies and the gain would be null.

In order to assess the impact of the environment effect on deterioration, on the cost gain, we consider Case 4 for which two sets of deterioration parameters are considered in the stressed environment. The evolution of the cost gain of the CI-based policy compared to the age-based policy depending on the stressed environment deterioration parameters and $C_{d}$ is presented in Figure 11.

One can notice that, when the impact of the environment on the deterioration increases, that is, $\left(\alpha_{2} \cdot \beta_{2}\right) /\left(\alpha_{1} \cdot \beta_{1}\right)$ increases, the gain increases. Actually, the decision making structures of the condition-based and the age-based maintenance policies does not take into account the future environment state (on the visibility horizon) unlike the predictive policies. Hence, when its impact increases, the gain resulting of taking it into account increases too.

For all the previous cases, the performance of the two predictive policies are almost the same although the prognostic is used in two different ways. Recall that the decision for both policies is not to choose any optimal time of replacement but an optimal one between the available opportunities. Therefore, since the predictive polices are close, the choice of the optimal opportunity would be the same and then the maintenance costs are the same. For that, we consider next, some cases for which the opportunities are closer on average so that the choice of the optimal opportunity can be different which would lead to different performances. Note that even when the two policies lead to the same maintenance cost, it is probably more convenient to use the CI-based policy in the considered configuration since, unlike the $R$-based policy, there are no parameters to optimize. If there is a change in the costs or the deterioration parameters, the risk threshold of the $R$-based policy should be optimized again while one can still apply the CI-based policy without any change.

Close Opportunities Case. Consider now closer opportunities, the distances between them are simulated using a uniform law $U(3,7)$. The results for two cases are presented in Table 1 . For both, $L=100,\left(\alpha_{1}, \beta_{1}\right)=(0.5,0.5),\left(\alpha_{2}, \beta_{2}\right)=(0.75,0.5)$, $C_{c}=100$, and $C_{p}=25$. Let $C_{\mathrm{CI}}$ and $C_{R}$ denote the expected long-run cost rate for, respectively, the CI-based and the $R$ based policies, MDTM $\mathrm{CI}_{\mathrm{CI}}$ and $\mathrm{MDTM}_{R}$ their mean distance to maintenance, and $G_{\text {cost }}$ and $G_{\mathrm{MDTM}}$ the gains in cost and in MDTM of the CI-based policy compared to the $R$-based policy.

For both cases, the CI-based policy is more performant than the $R$-based policy in terms of cost saving (around 2.4\%) and mean distance to maintenance which is slightly higher for the CI-based policy. However, the performance difference is not considerable.

Opportunities with Different Maintenance Costs. Recall that the main difference between the two policies is that the $R$ based policy uses only the prognostic on the conditional reliability while the CI-based policy uses the prognostic but also the different maintenance costs. If these costs are different depending on the maintenance opportunity the CIbased policy should probably be more efficient. In order to study this configuration, we examined several cases for which costs are different depending on the opportunity, some are presented in Table 2. For the studied Cases (7 to 11) we simulated the environment changes, the distance between opportunities and the corrective $\operatorname{cost} C_{c}$ with uniform laws, $L=100,\left(\alpha_{1}, \beta_{1}\right)=(0.5,0.5)$ and $\left(\alpha_{2}, \beta_{2}\right)=(0.75,0.5)$. $C_{p} / C_{c}$ and $C_{d} / C_{c}$ are fixed for each case since the variability of these rates affect the performance of the policies, in this case we cannot examine clearly the effect of other parameters. 
Let $C_{p i}, C_{c i}$, and $C_{d i}$ denote the preventive, corrective, and downtime costs for the first opportunity, and $C_{p(i+1)}, C_{c(i+1)}$ and $C_{d(i+1)}$ the costs for the second one. The expressions of the cost indicators are

$$
\begin{aligned}
& \mathrm{CI}_{i}=\frac{C_{p i}}{t_{i}} \\
\mathrm{CI}_{i+1}=\frac{1}{t_{i+1}+I_{1}+I_{2}} & \left(C_{p(i+1)} \cdot R\left(t_{i+1} \mid X\left(t_{i}\right)=x_{0}\right)\right. \\
& +C_{c(i+1)} \cdot F\left(t_{i+1} \mid X\left(t_{i}\right)=x_{0}\right) \\
& -I_{1} \cdot C_{d i}+I_{2} \cdot C_{d(i+1)} \\
& +\bar{t} \cdot F\left(\bar{t} \mid X\left(t_{i}\right)=x_{0}\right) \\
& \left.\cdot\left(C_{d i}-C_{d(i+1)}\right)\right),
\end{aligned}
$$

where $\bar{t}=\left(t_{i}+t_{i+1}\right) / 2$.

Contrary to what was expected, the $R$-based policy is more efficient in some cases ( 7 to 10) than the CI-based policy (Table 2). Actually, in the first part of the life cycle of the system, the probability of failure is almost null; a maintenance operation at any opportunity would be a preventive replacement. Hence, $C_{i+1} \rightarrow C_{p(i+1)} / t_{i+1}$. In the case where the opportunities are relatively close and the cost is relatively variable, the cost indicators at the first part of the life cycle can be as follows: $C_{i}=C_{p i} / t_{i}<C_{i+1} \rightarrow C_{p(i+1)} / t_{i+1}$. In this case a preventive replacement will be scheduled at the first opportunity using the CI-based policy. Since the probability of failure is almost null, no maintenance action would be planned using the $R$-based policy which is a better decision in this case. Note that the cost indicators on later opportunities $(i+2)$ or $(i+3)$, for example, would be lower than $\mathrm{CI}_{i}$. Hence, if more opportunities are known on the visible horizon, a better decision can be taken. One can note that the difference in performance is lower in Case 8 than in Case 7 since the opportunities are more spaced, and this difference decreases with the decrease of the costs variability (Case 9). For Cases 10 and 11, the opportunities are sufficiently spaced relatively to the variability of the costs; the policies performance is hence similar.

\section{Conclusion}

This paper deals with a maintenance study taking into account jointly two main problems: (i) the choice of coherent maintenance structure that takes into account specific constraints related to maintenance opportunities on a finite rolling horizon, and (ii) the choice of the information used in the decision, and the best way to integrate it. For that purpose, we considered four maintenance strategies corresponding to the age-based, condition-based, and predictive maintenance approaches. We compared the policies performance mainly on the basis of a cost criterion given the considered economic context. The studied cases proved that the developed policies within the predictive approach lead to cost saving comparing to the condition-based and the age-based policies. This result was expected since the predictive approach integrate more "useful" information into the decision making structure. However, the carried out study allowed to assess the impact of the different parameters on the different policies. In particular, the study permitted to propose and compare two ways of using the prognostic in the developed predictive policies and to decide the best way to use the information according to the case.

Beyond the considered context and cases, it is clear that the type of information and the way to use it decide directly the quality of the decision making. However, one should always compare the cost of the information (monitoring equipments for example and data collecting) with the cost saving to decide which strategy leads to the best performance.

\section{Future Work}

In this work, the decision is taken when the system reaches the first opportunity. In practice, it might be more realistic to consider that scheduling a preventive operation should be done at least before a given duration/distance. In this case, the computation of the decision indicators is slightly different than the one presented in this work. However, the results and conclusions should be similar.

In this study, the environment changes and distances between opportunities were simulated with different laws according to the considered case. The visibility horizon was supposed to contain (at least) two maintenance opportunities. It would be interesting to fix the length of the visibility horizon and thus have one or more visible opportunities. In this case, the decision making structure should be adapted. When only one opportunity is visible, the cost indicator CI can be compared to a cost threshold (to be optimized) if one wants to apply the CI-based policy, for example. The application of the age-based, condition-based, and $R$-based policies does not change.

\section{Acknowledgments}

This work is supported by the European project MoDeMaintenance on Demand (http://fp7-mode.eu/) funded from the European Community's 7th Framework Program (Project SCP8-GA-2009-233890-FP7 Sustainable Surface Transport).

\section{References}

[1] A. K. S. Jardine, D. Lin, and D. Banjevic, "A review on machinery diagnostics and prognostics implementing condition-based maintenance," Mechanical Systems and Signal Processing, vol. 20, no. 7, pp. 1483-1510, 2006.

[2] O. Dragomir, R. Gouriveau, F. Dragomir, E. Minca, and N. Zerhouni, "Review of prognostic problem in condition-based maintenance," in Proceedings of the European Control Conference (ECC '09), pp. 1585-1592, 2009.

[3] Y. Peng, M. Dong, and M. J. Zuo, "Current status of machine prognostics in condition-based maintenance: a review," International Journal of Advanced Manufacturing Technology, vol. 50, no. 1-4, pp. 297-313, 2010.

[4] N. Gorjian, L. Ma, M. Mittinty, P. Yarlagadda, and Y. Sun, "A review on degradation models in reliability analysis," in 
Proceedings of the 4th World Congress on Engineering Asset Management, vol. 28, 2009.

[5] E. Khoury, E. Deloux, A. Grall, and C. Bérenguer, "On the use of time-limited and monitoring information for maintenance decision support," in Proceedings of 8th IFAC Symposium on Fault Detection, Supervision and Safety of Technical Processes (SAFEPROCESS '12), pp. 480-485, 2012.

[6] E. Khoury, Modélisation de la Durée de Vie résiduelle et Maintenance Prédictive: Application à des Véhicules Industriels [Ph.D. thesis], Université de technologie de Troyes, Troyes, France, 2012.

[7] M. Rausand and A. Høyland, System Reliability Theory: Models, Statistical Methods and Applications, John Wiley \& Sons, Hoboken, NJ, USA, 2nd edition, 2004.

[8] I. Gertsbakh, Reliability Theory, Springer, Berlin, Germany, 2000.

[9] E. Deloux, B. Castanier, and C. Bérenguer, "Predictive maintenance policy for a gradually deteriorating system subject to stress," Reliability Engineering and System Safety, vol. 94, no. 2, pp. 418-431, 2009.

[10] X. Zhao, M. Fouladirad, C. Bérenguer, and L. Bordes, "Condition-based inspection/replacement policies for nonmonotone deteriorating systems with environmental covariates," Reliability Engineering and System Safety, vol. 95, no. 8, pp. 921-934, 2010.

[11] A. Ponchet, M. Fouladirad, and A. Grall, "Assessment of a maintenance model for a multi-deteriorating mode system," Reliability Engineering and System Safety, vol. 95, no. 11, pp. 1244-1254, 2010.

[12] X.-S. Si, W. Wang, C.-H. Hu, and D.-H. Zhou, "Remaining useful life estimation: a review on the statistical data driven approaches," European Journal of Operational Research, vol. 213, no. 1, pp. 1-14, 2011.

[13] J. Z. Sikorska, M. Hodkiewicz, and L. Ma, "Prognostic modelling options for remaining useful life estimation by industry," Mechanical Systems and Signal Processing, vol. 25, no. 5, pp. 1803-1836, 2011.

[14] A. Grall, L. Dieulle, C. Bérenguer, and M. Roussignol, "Continuous-time predictive-maintenance scheduling for a deteriorating system," IEEE Transactions on Reliability, vol. 51, no. 2, pp. 141-150, 2002.

[15] C. T. Barker and M. J. Newby, "Optimal non-periodic inspection for a multivariate degradation model," Reliability Engineering and System Safety, vol. 94, no. 1, pp. 33-43, 2009.

[16] M. Abdel-Hameed, "A gamma wear process," IEEE Transactions on Reliability, vol. R-24, no. 2, pp. 152-153, 1975.

[17] E. Khoury, A. Grall, and C. Bérenguer, "A comparison of rul-based and deterioration-based maintenance policies for gradually deteriorating systems," in Reliability, Risk and SafetyBack To the Future, Ale, B. J. M. Ale, I. A. Papazoglou, and E. Zio, Eds., pp. 530-537, 2010.

[18] J. M. van Noortwijk, "A survey of the application of gamma processes in maintenance," Reliability Engineering and System Safety, vol. 94, no. 1, pp. 2-21, 2009.

[19] E. Deloux, B. Castanier, and C. Bérenguer, "Maintenance policy for a deteriorating system evolving in a stressful environment," Proceedings of the Institution of Mechanical Engineers $O$, vol. 222, no. 4, pp. 613-622, 2008.

[20] H. Peng, Q. Feng, and D. W. Coit, "Reliability and maintenance modeling for systems subject to multiple dependent competing failure processes," IIE Transactions, vol. 43, no. 1, pp. 12-22, 2011.
[21] J. Lawless and M. Crowder, "Covariates and random effects in a gamma process model with application to degradation and failure," Lifetime Data Analysis, vol. 10, no. 3, pp. 213-227, 2004.

[22] N. Gebraeel and J. Pan, "Prognostic degradation models for computing and updating residual life distributions in a timevarying environment," IEEE Transactions on Reliability, vol. 57, no. 4, pp. 539-550, 2008.

[23] C. J. $\mathrm{Lu}$ and W. Q. Meeker, "Using degradation measures to estimate a time-to-failure distribution," Technometrics, vol. 35, no. 2, pp. 161-174, 1993.

[24] A. Lehmann, "Joint modeling of degradation and failure time data," Journal of Statistical Planning and Inference, vol. 139, no. 5, pp. 1693-1706, 2009.

[25] R. Dekker and M. Dijkstra, "Opportunity-based age replacement: exponentially distributed times between opportunities," Naval Research Logistics, vol. 39, no. 2, pp. 175-190, 1992.

[26] H. Wang, "A survey of maintenance policies of deteriorating systems," European Journal of Operational Research, vol. 139, no. 3, pp. 469-489, 2002.

[27] R. Ahmad and S. Kamaruddin, "An overview of time-based and condition-based maintenance in industrial application," Computers and Industrial Engineering, vol. 63, no. 1, pp. 135-149, 2012.

[28] H. Pham and H. Wang, "Imperfect maintenance," European Journal of Operational Research, vol. 94, no. 3, pp. 425-438, 1996. 


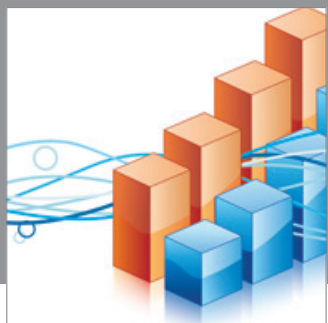

Advances in

Operations Research

mansans

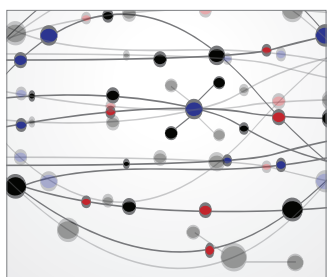

The Scientific World Journal
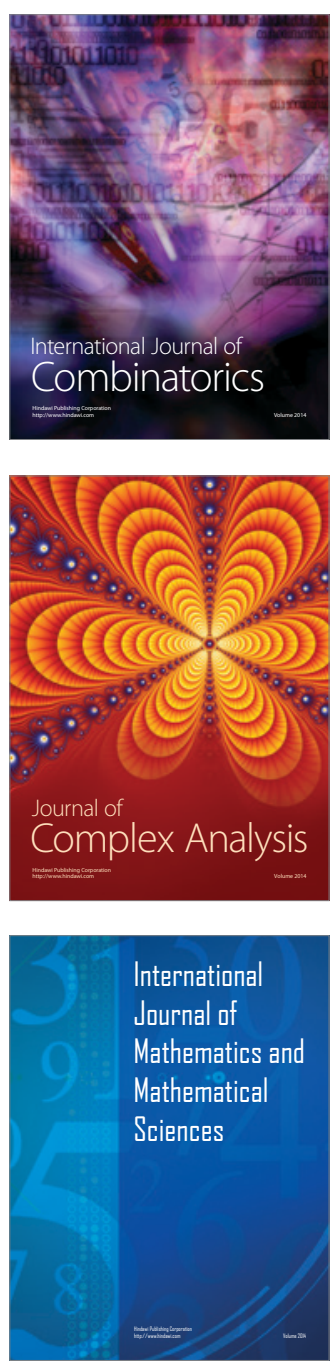
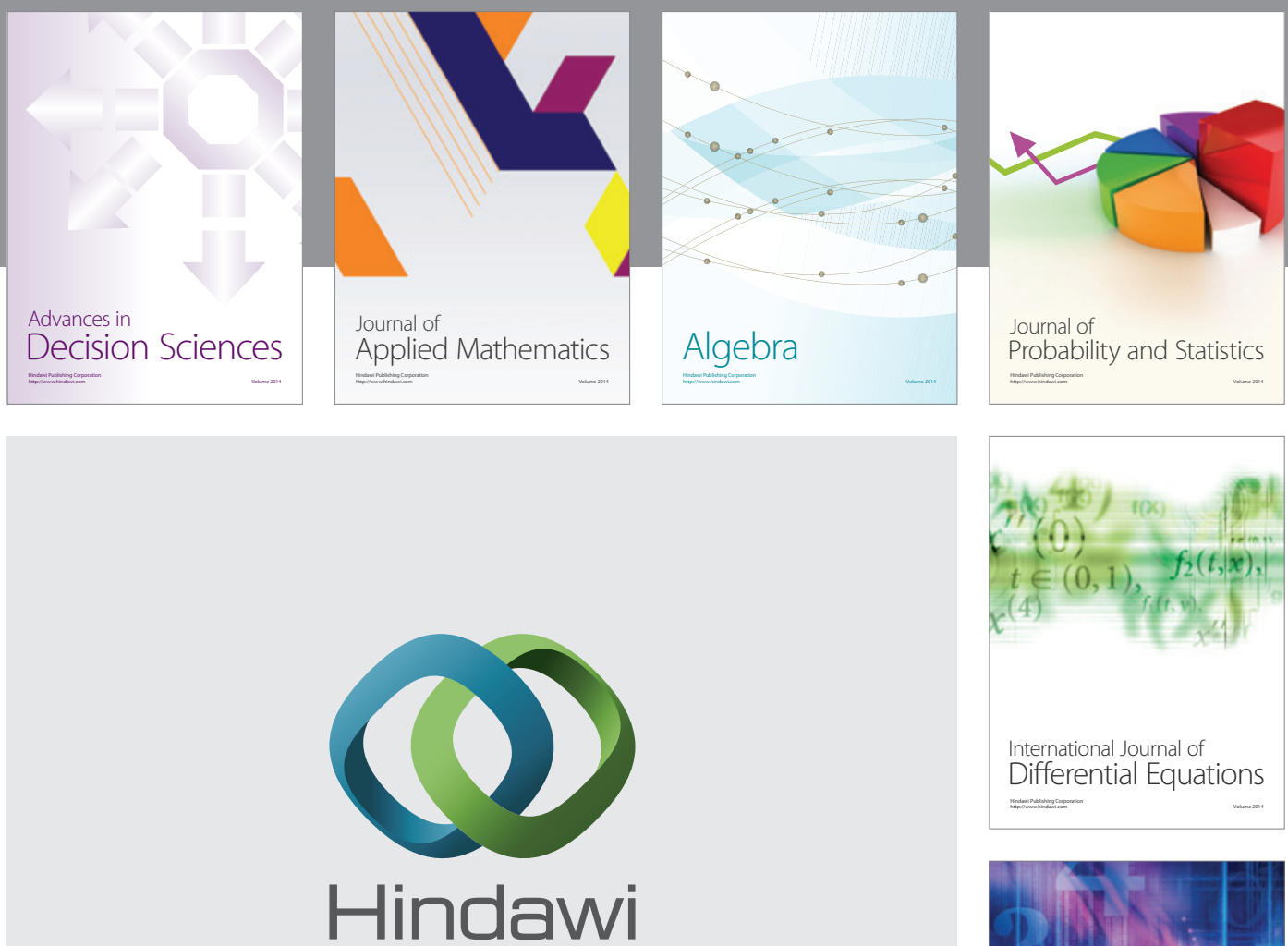

Submit your manuscripts at http://www.hindawi.com
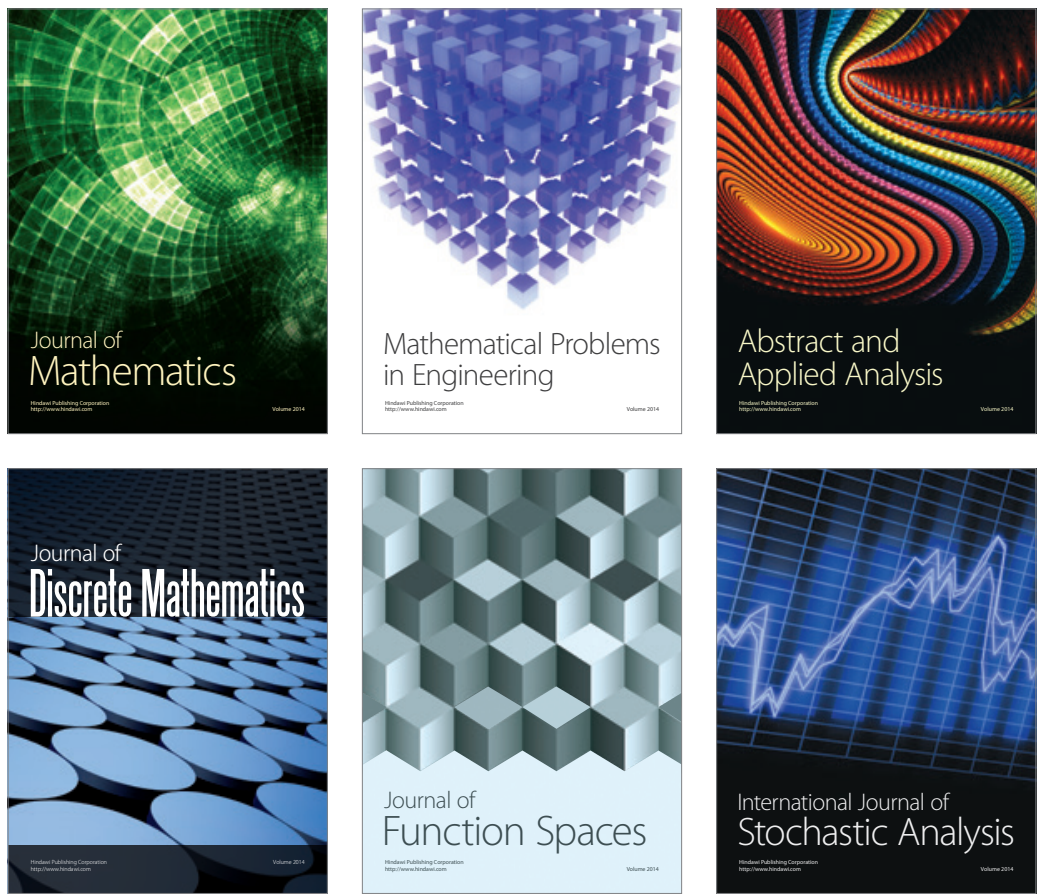

Journal of

Function Spaces

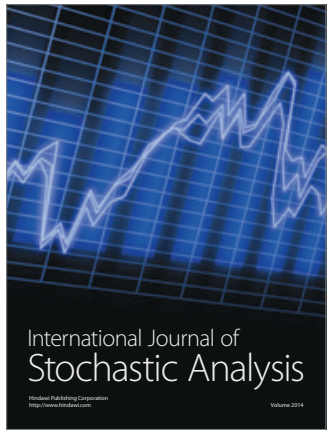

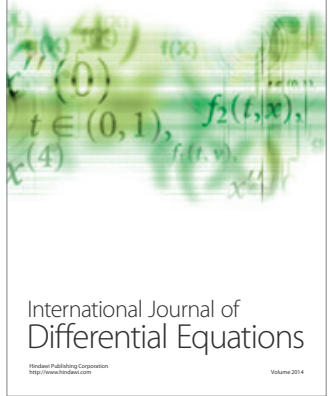
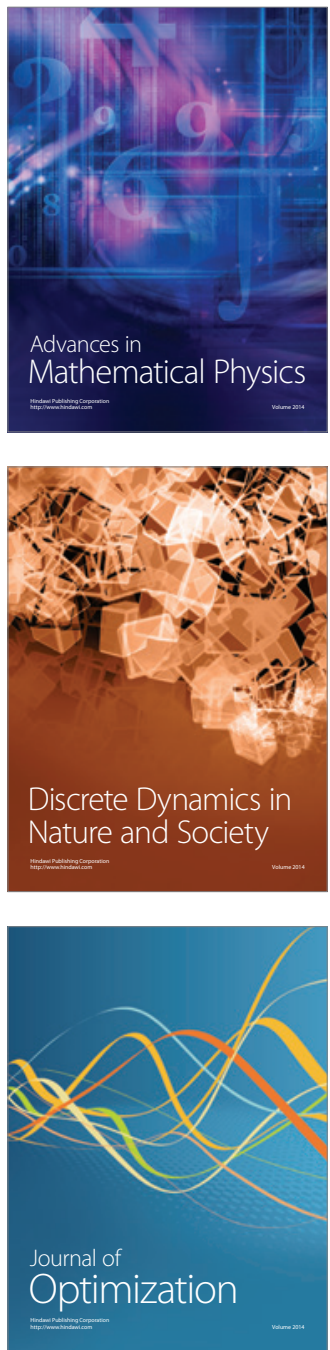